\title{
Centering inclusivity in the design of online conferences - An OHBM - Open Science perspective
}

Elizabeth Levitis ${ }^{\star 1,2}$, Cassandra D Gould van Praag ${ }^{\star \# 3,4}$, Rémi Gau ${ }^{5}$, Stephan Heunis ${ }^{\# 6}$, Elizabeth DuPre ${ }^{7}$, Gregory Kiar ${ }^{8}$, Katherine L Bottenhorn ${ }^{9}$, Tristan Glatard ${ }^{10}$, Aki Nikolaidis ${ }^{11}$, Kirstie Jane Whitaker ${ }^{12}$, Matteo Mancini ${ }^{13,14,15}$, Guiomar Niso ${ }^{16,17}$, Soroosh Afyouni ${ }^{18}$, Eva Alonso-Ortiz ${ }^{19}$, Stefan Appelhoff ${ }^{20}$, Aurina Arnatkeviciute $^{21}$, Selim Melvin Atay ${ }^{22}$, Tibor Auer ${ }^{23}$, Giulia Baracchini ${ }^{24,25}$, Johanna M. M. Bayer ${ }^{26,27}$, Michael J.S. Beauvais ${ }^{28}$, Janine D Bijsterbosch ${ }^{29}$, Isil P Bilgin ${ }^{30}$, Saskia Bollmann ${ }^{31}$, Steffen Bollmann ${ }^{32,33}$, Rotem Botvinik-Nezer ${ }^{34}$, Molly G Bright ${ }^{35,36}$, Vince D Calhoun ${ }^{37}$, Xiao Chen ${ }^{38,39,40}$, Sidhant Chopra ${ }^{41}$, Hu Chuan-Peng ${ }^{42}$, Thomas G. Close ${ }^{43,44}$, Savannah L Cookson ${ }^{45}$, R. Cameron Craddock ${ }^{46}$, Alejandro De La Vega $^{47}$, Benjamin De Leener ${ }^{48,49}$, Damion V Demeter ${ }^{50}$, Paola Di Maio ${ }^{51,52}$, Erin W Dickie ${ }^{53,54}$, Simon B Eickhoff ${ }^{55,56}$, Oscar Esteban ${ }^{57}$, Karolina Finc ${ }^{58}$, Matteo Frigo ${ }^{59,60}$, Saampras Ganesan ${ }^{61,62}$, Melanie Ganz $z^{63,64}$, Kelly G Garner ${ }^{65,66,67}$, Eduardo A Garza-Villarreal ${ }^{68}$, Gabriel Gonzalez-Escamilla ${ }^{69}$, Rohit Goswami ${ }^{70,71}$, John D Griffiths ${ }^{72,73}$, Tijl Grootswagers ${ }^{74}$, Samuel Guay ${ }^{75}$, Olivia Guest ${ }^{76}$, Daniel A Handwerker ${ }^{77}$, Peer Herholz ${ }^{78}$, Katja Heuer ${ }^{79,80}$, Dorien C. Huijser ${ }^{81,82}$, Vittorio lacovella ${ }^{83}$, Michael JE Joseph ${ }^{84}$, Agah Karakuzu ${ }^{85,86}$, David B Keator $^{87}$, Xenia Kobeleva ${ }^{88,89}$, Manoj Kumar ${ }^{90}$, Angela R. Laird ${ }^{91}$, Linda J. Larson-Prior ${ }^{92,93,94}$, Alexandra Lautarescu $^{95,96}$, Alberto Lazari ${ }^{97}$, Jon Haitz Legarreta ${ }^{98}$, Xue-Ying Li ${ }^{99,100,101,102}$, Jinglei Lv ${ }^{103}$, Sina Mansour L. ${ }^{61,62}$, David Meunier ${ }^{104}$, Dustin Moraczewski ${ }^{105}$, Tulika Nandi ${ }^{106}$, Samuel A Nastase ${ }^{107}$, Matthias Nau ${ }^{108,109}$, Stephanie Noble ${ }^{110}$, Martin Norgaard ${ }^{111,112}$, Johnes Obungoloch ${ }^{113}$, Robert Oostenveld ${ }^{114,115}$, Edwina $R$ Orchard ${ }^{116}$, Ana Luísa Pinho ${ }^{117}$, Russell A Poldrack ${ }^{118}$, Anqi Qiu ${ }^{119,120}$, Pradeep Reddy Raamana ${ }^{121}$, Ariel Rokem $^{122}$, Saige Rutherford ${ }^{123,124}$, Malvika Sharan ${ }^{12}$, Thomas B Shaw ${ }^{125}$, Warda T Syeda ${ }^{126}$, Meghan M Testerman $^{127}$, Roberto Toro ${ }^{128,79}$, Sofie L Valk ${ }^{130,131}$, Sofie Van Den Bossche ${ }^{132}$, Gaël Varoquaux ${ }^{117,133}$, František Váša ${ }^{134}$, Michele Veldsman ${ }^{135}$, Jakub Vohryzek ${ }^{4,136}$, Adina S. Wagner ${ }^{131}$, Reubs J Walsh ${ }^{137,138}$, Tonya White ${ }^{139,140}$, Fu-Te Wong ${ }^{141,142}$, Xihe Xie ${ }^{143,144}$, Chao-Gan Yan ${ }^{145,146,147}$, Yu-Fang Yang ${ }^{148}$, Yohan Yee ${ }^{149,150}$, Gaston E Zanitti ${ }^{117}$, Ana E Van Gulick ${ }^{151,152}$, Eugene Duff ${ }^{153,3}$, Camille Maumet ${ }^{154+}$

* Joint first authorship, = OHBM Brainhack 2020 co-chairs, " Open Science Room 2020 co-chairs,

+ corresponding author

1 Section on Developmental Neurogenomics, National Institute of Mental Health, Bethesda, MD, USA

2 Centre for Medical Image Computing, Department of Computer Science, University College London, London, UK

3 Wellcome Centre for Integrative Neuroimaging, University of Oxford, Oxford, UK

4 Department of Psychiatry, University of Oxford, Oxford, UK

5 Institute of Psychology, Université catholique de Louvain, Louvain la neuve, Belgium

6 Department of Electrical Engineering, Eindhoven University of Technology, Eindhoven, The Netherlands

7 NeuroDataScience - ORIGAMI laboratory, McGill University, Montreal, Canada

8 Department of Biomedical Engineering, McGill University, Montreal, Canada

9 Department of Psychology, Florida International University, Miami, FL, USA

10 Department of Computer Science and Software Engineering, Concordia University, Montreal, Canada

11 Center for the Developing Brain, The Child Mind Institute, New York City, NY, USA

12 The Alan Turing Institute, London, UK

13 Department of Neuroscience, Brighton and Sussex Medical School, University of Sussex, Brighton, UK

14 Cardiff University Brain Research Imaging Centre, Cardiff University, Cardiff, UK

15 NeuroPoly Lab, Polytechnique Montreal, Montreal, Canada

16, Indiana University, Bloomington, USA

17 Universidad Politécnica de Madrid and CIBER-BBN, Madrid, Spain

18 Big Data Institute, University of Oxford, Oxford, UK

19 Electrical Engineering, Polytechnique Montréal, Montréal, Canada

20 Center for Adaptive Rationality, Max Planck Institute for Human Development, Berlin, Germany

21 The Turner Institute for Brain and Mental Health, School of Psychological Sciences, and Monash Biomedical Imaging, Monash University, Victoria, Australia

22 Neuroscience and Neurotechnology, Middle East Technical University, Ankara, Turkey

23 School of Psychology, University of Surrey, Guildford, UK

24 Department of Neurology and Neurosurgery, McGill University, Montréal, Canada

25 Montréal Neurological Institute, Montréal, Canada

26 Centre for Youth Mental Health, University of Melbourne, Melbourne, Australia

27 Orygen Youth Health, Melbourne, Australia

28 Centre of Genomics and Policy, McGill University, Montreal, Canada

29 Department of Radiology, Washington University School of Medicine, Saint Louis, MO, USA

30 Department of Biomedical Engineering, Cybernetics, The School of Biological Sciences, The University of Reading, Reading, UK

31 Centre for Advanced Imaging, The University of Queensland, Brisbane, Australia

32 School of Information Technology and Electrical Engineering, The University of Queensland, Brisbane, Australia

33 ARC Training Centre for Innovation in Biomedical Imaging Technology, The University of Queensland, Brisbane, Australia

34 Department of Psychological and Brain Sciences, Dartmouth College, Hanover, NH, USA

35 Physical Therapy and Human Movement Sciences, Feinberg School of Medicine, Northwestern University, Chicago, IL, USA

36 Biomedical Engineering, McCormick School of Engineering and Applied Sciences, Northwestern University, Evanston, IL, USA

37 Tri-institutional Center for Translational Research in Neuroimaging and Data Science (TReNDS), Georgia State, Georgia Tech,

Emory, Atlanta, USA

38 CAS Key Laboratory of Behavioral Science, Institute of Psychology, Beijing, China 
39 Magnetic Resonance Imaging Research Center, Institute of Psychology, Chinese Academy of Sciences, Beijing, China 40 International Big-Data Center for Depression Research, Chinese Academy of Sciences, Beijing, China

41 Turner Institute for Brain and Mental Health, Monash University, Melbourne, Australia.

42 School of Psychology, Nanjing Normal University, Nanjing, China

43 Department of Biomedical Engineering, The University of Sydney, Sydney, Australia

44 National Imaging Facility, The University of Sydney, Sydney, Australia

45 Helen Wills Neuroscience Institute, University of California, Berkeley, Berkeley, CA, USA

46 Department of Diagnostic Medicine, The University of Texas at Austin Dell Medical School, Austin Texas

47 Department of Psychology, The University of Texas at Austin, Austin, TX, US

48 Department of Computer and Software Engineering, Polytechnique Montreal, Montreal, Quebec, Canada

49 Research Centre, Sainte-Justine University Hospital Center, Montreal, Quebec, Canada

50 Department of Psychology, The University of Texas at Austin, Austin, TX USA

51 Center for Systems, Knowledge Representation and Neuroscience, Edinburgh and Taipei, UK and Taiwan

52 Institute for Globally Distributed Open Research and Education (IGDORE),

$53 \mathrm{Krembil}$ Centre for Neuroinformatics, Centre for Addiction and Mental Health, Toronto, Canada

54 Dept. of Psychiatry, University of Toronto, Toronto, Canada,

55 Institute of Systems Neuroscience, Medical Faculty, Heinrich Heine University Düsseldorf, Düsseldorf, Germany

56 Institute of Neuroscience and Medicine, Brain \& Behaviour (INM-7), Research Centre Jülich, Jülich, Germany

57 Dept. of Radiology, Lausanne University Hospital and University of Lausanne, Lausanne, Switzerland

58 Centre for Modern Interdisciplinary Technologies, Nicolaus Copernicus University, Toruń, Poland

59 Athena Project Team, Inria Sophia Antipolis - Méditerranée, Valbonne, France

60 Université Côte d'Azur, Nice, France

61 Melbourne Neuropsychiatry Centre, The University of Melbourne, Melbourne, Australia

62 Department of Biomedical Engineering, The University of Melbourne, Melbourne, Australia

63 Neurobiology Research Unit, Rigshospitalet, Copenhagen, Denmark

64 Department of Computer Science, University of Copenhagen, Copenhagen, Denmark

65 Queensland Brain Institute, University of Queensland, St. Lucia, Australia

66 School of Psychology, University of Birmingham, Birmingham, UK

67 School of Psychology, University of Queensland, St. Lucia, Australia

68 Instituto de Neurobiologia, Universidad Nacional Autonoma de Mexico campus Juriquilla, Queretaro, Mexico

69 Department of Neurology, Focus Program Translational Neuroscience (FTN), Rhine Main Neuroscience Network (rmn2), University

Medical Center of the Johannes Gutenberg University Mainz, Mainz, Germany

70 Faculty of Physical Sciences, University of Iceland, Reykjavik, Iceland

71 Department of Chemistry, Indian Institute of Technology Kanpur, Kanpur, India

72 Department of Psychiatry, University of Toronto, Toronto, Canada

73 Krembil Centre for Neuroinformatics, Centre for Addiction and Mental Health, Toronto, Canada

74 The MARCS Institute for Brain, Behaviour \& Development, Western Sydney University, Sydney, Australia

75 Psychology, Université de Montréal, Montreal, Canada

76 Donders Centre for Cognitive Neuroimaging, Radboud University, Nijmegen, Netherlands

77 Section on Functional Imaging Methods, Laboratory of Brain and Cognition, National Institute of Mental Health, Bethesda, MD, USA

78 NeuroDataScience - ORIGAMI laboratory, McConnell Brain Imaging Centre, The Neuro (Montreal Neurological Institute-Hospital),

Faculty of Medicine, McGill University, Montreal, Canada

79 Center for Research and Interdisciplinarity (CRI), INSERM U1284, Université de Paris, Paris, France

80 Max Planck Institute for Human Cognitive and Brain Sciences, Leipzig, Germany

81 Erasmus School of Social and Behavioral Sciences, Erasmus University Rotterdam, Rotterdam, the Netherlands

82 Developmental and Educational Psychology, Leiden University, Leiden, the Netherlands

83 Center for Mind, Brain Sciences - CIMeC, University of Trento, Rovereto, Italy

84 Campbell Family Mental Health Research Institute, Centre for Addiction and Mental Health, Toronto, Canada

85 NeuroPoly Lab, Institute of Biomedical Engineering, Polytechnique Montréal, Montréal, Canada

86 Montréal Heart Institute, University of Montréal, Montréal, Canada

87 Psychiatry and Human Behavior, University of California, Irvine, Irvine, USA

88 Department of Neurology, University Hospital Bonn, Bonn, Germany

89, German Center for Neurodegenerative Diseases (DZNE), Bonn, Germany

90 Princeton Neuroscience Institute, Princeton University, Princeton, NJ, USA

91 Department of Physics, Florida International University, Miami, USA

92 Psychiatric Research Institute, University of Arkansas for Medical Sciences, Little Rock, Arkansas, USA

93 Arkansas Children's Nutrition Center, Little Rock AR, USA

94 Dept Neurology, Pediatrics, Neuroscience \& Developmental Sciences, Biomedical Informatics, University of Arkansas for Medical Sciences, Little Rock AR, USA

95 Department of Perinatal Imaging and Health, Centre for the Developing Brain, School of Biomedical Engineering and Imaging Sciences, King's College London, London, UK

96 Department of Forensic and Neurodevelopmental Sciences, Institute of Psychiatry, Psychology and Neuroscience, King's College London, London, UK

97 Wellcome Centre for Integrative Neuroimaging, FMRIB, Nuffield Department of Clinical Neurosciences, University of Oxford, UK

98 Department of Computer Science, Université de Sherbrooke, Sherbrooke, Canada

99 Sino-Danish College, University of Chinese Academy of Sciences, Beijing, China

100 CAS Key Laboratory of Behavioral Science, Institute of Psychology, Beijing, China

101 Sino-Danish Center for Education and Research, Beijing, China

102 CFIN and PET Center, Aarhus University, Aarhus, Denmark

103 School of Biomedical Engineering \& Brain and Mind Center, University of Sydney, Sydney, NSW AU.

104 Aix Marseille Univ, CNRS, INT, Inst Neurosci Timone, Marseille, France

105 National Institute of Mental Health, Bethesda, MD, USA

106 Nuffield Department of Clinical Neurosciences, University of Oxford, Oxford, UK

107 Princeton Neuroscience Institute, Princeton University, Princeton, NJ, USA

108 Section on Learning and Plasticity, Laboratory of Brain and Cognition, National Institute of Mental Health, Bethesda, MD, USA

109 Max-Planck-Institute for Human Cognitive and Brain Sciences, Leipzig, Germany 
112 Neurobiology Research Unit, Department of Neurology, Rigshospitalet, Copenhagen University Hospital, Copenhagen, Denmark

113 Department of Biomedical Sciences and Engineering, Mbarara University of Science and Technology, Mbarara City, Uganda

114 Donders Institute for Brain, Cognition and Behaviour, Radboud University, Nijmegen, The Netherlands

115 NatMEG, Karolinska Institutet, Stockholm, Sweden

116 Turner Institute of Brain and Mental Health, Monash University, Melbourne, Australia

117 Université Paris-Saclay, Inria, CEA, Palaiseau, France

118 Department of Psychology, Stanford University, Stanford, CA, USA

119 Department of Biomedical Engineering, National University of Singapore, Singapore, Singapore, The N.1 Institute for Health, National University of Singapore, Singapore, Singapore, Smart Systems Institute, National University of Singapore, Singapore, Singapore

120 Department of Biomedical Engineering, Johns Hopkins University, Baltimore, MD, USA

121 Department of Radiology, University of Pittsburgh, Pittsburgh, PA, USA

122 Department of Psychology \& eScience Institute, University of Washington, Seattle, WA, USA

123 Department of Cognitive Neuroscience, Radboud University Medical Center, Nijmegen, the Netherlands

124 Department of Psychiatry, University of Michigan, Ann Arbor, MI, USA

125 Centre for Advanced Imaging, The University of Queensland, Brisbane, Australia

126 Melbourne Neuropsychiatry Centre, Department of Psychiatry, The University of Melbourne, Victoria, Australia

127 University Library, Princeton University, Princeton, USA

128 Neuroscience department, Institut Pasteur, Paris, France

130 Otto Hahn Group Cognitive Neurogenetics, Max Planck Institute for Human Cognitive and Brain Sciences, Leipzig, Germany

131 Institute of Neuroscience and Medicine Brain \& Behaviour (INM-7), Research Center Jülich, Jülich, Germany

132 Department of Data Analysis, Faculty of Psychology and Educational Sciences, Ghent University, Belgium

133 Montreal Neurological Institute, McGill, Montreal, Canada

134 Department of Neuroimaging, Institute of Psychiatry Psychology \& Neuroscience, King's College London, London, UK

135 Department of Experimental Psychology, University of Oxford, Oxfordshire, UK

136 Center for Music in the Brain, Department of Clinical Medicine, Aarhus University, Aarhus, Denmark

137 Department of Clinical, Neuro-, and Developmental Psychology, Vrije Universiteit Amsterdam, Amsterdam, Netherlands

138 Center for Applied Transgender Studies

139 Department of Child and Adolescent Psychiatry, Erasmus University Medical Centre, Rotterdam, Netherlands

140 Department of Radiology and Nuclear Medicine, Erasmus University Medical Centre, Rotterdam, Netherlands

141 Institute of Linguistics, Academia Sinica, Taipei, Taiwan

142 Institute of Medical Science, University of Toronto, Toronto, Canada

143 Department of Neuroscience, Weill Cornell Graduate School, New York City, U.S.A.

144 Department of Radiology and BioEngineering, University of California San Francisco, San Francisco, U.S.A.

145 CAS Key Laboratory of Behavioral Science, Institute of Psychology, Beijing, China

146 Department of Psychology, University of Chinese Academy of Sciences, Beijing, China

147 International Big-Data Center for Depression Research, Chinese Academy of Sciences, Beijing, China

148 Department of Psychology, University of Würzburg, Würzburg, Germany

149 Department of Medical Biophysics, University of Toronto, Toronto, Canada

150 Mouse Imaging Centre, The Hospital for Sick Children, Toronto, Canada

151 Figshare, Cambridge, MA, USA

152 University Libraries, Carnegie Mellon University, Pittsburgh, PA, USA

153 Department of Paediatrics, University of Oxford, Oxford, UK

154 Inria, Univ Rennes, CNRS, Inserm, IRISA UMR 6074, Empenn ERL U 1228, Rennes, France

\section{Abstract}

As the global health crisis unfolded throughout the world, many academic conferences moved online in 2020 .

This move has been hailed as a positive step towards inclusivity in its attenuation of economic, physical and

legal barriers and effectively enabled many individuals who have traditionally been underrepresented to join

and participate. A number of studies have outlined how moving online made it possible to gather a more

global community and has increased opportunities for individuals with various constraints, e.g. caregiving responsibilities.

Yet, the mere existence of online conferences is unfortunately no guarantee that everyone can attend and participate meaningfully. In fact, many elements of an online conference are still significant barriers to truly diverse participation: the tools used can be inaccessible for some individuals; the scheduling choices can favour some geographical locations; the setup of the conference can provide more visibility to 
well-established researchers and reduce opportunities for early career researchers. While acknowledging the benefits of an online setting, especially for individuals who have traditionally been underrepresented or excluded, we recognize that fostering social justice requires inclusivity to actively be centered in every aspect of online conference design.

Here, we draw from the literature and from our own experiences to identify practices that purposefully encourage a diverse community to: attend, participate in, and lead online conferences. Reflecting on how to design more inclusive online events is especially important as multiple scientific organizations have announced that they will continue offering an online version of their event when in-person conferences can resume.

\section{Keywords}

Online conferences, Diversity, Inclusivity, Open science, Collaborative events.

\section{Background}

Many of the conferences scheduled for 2020 had to move online in a rush as the global health crisis unfolded. By removing the need to travel, obtain visas, and by decreasing overall costs, this move enabled more individuals who have traditionally been underrepresented to not only participate in $[1,2]$ but to lead conferences [3]. This shift to online events has considerably changed how researchers envision conferences and will likely have long-lasting consequences in research communities.

Although online conferences suggest a more inclusive experience than in-person events, their mere existence is no guarantee that everyone can attend and participate meaningfully [4]. Some issues present at in-person events are in fact accentuated in online events: with an increased focus on talks, the visibility gained by early career researchers presenting posters may be reduced. Some challenges are unique to online events, such as the scheduling of sessions to accommodate a range of time zones and participant commitments; or the availability of internet connectivity to seamlessly stream event content and participate in live sessions. Still other concerns exist across both conference structures and reflect broader long-standing problems in STEM, such as minorities being underrepresented in leadership positions [5].

These issues were likely to have been even more pronounced in the 2020 events as organizing committees had to adapt and make quick decisions. In practice, this meant that organizers often made this transition without enough time to carefully cater to attendees with different needs from their own, seek community input, onboard volunteers, and to promote their events beyond their own circles. 
Now is a good time to review these issues and identify best practices for designing intentionally inclusive online events. Here, we focus on inclusivity, the process to purposefully encourage a diverse community to join, actively take part and lead online conferences. While there is no one-size-fits-all solution, we consider diversity with respect to traditionally underrepresented groups, including: individuals from low and middle income countries; those with caretaker responsibilities; those with disabilities, chronic illness, neurodiversity or mental health issues; students and early career researchers.

\section{Towards more inclusive online conferences}

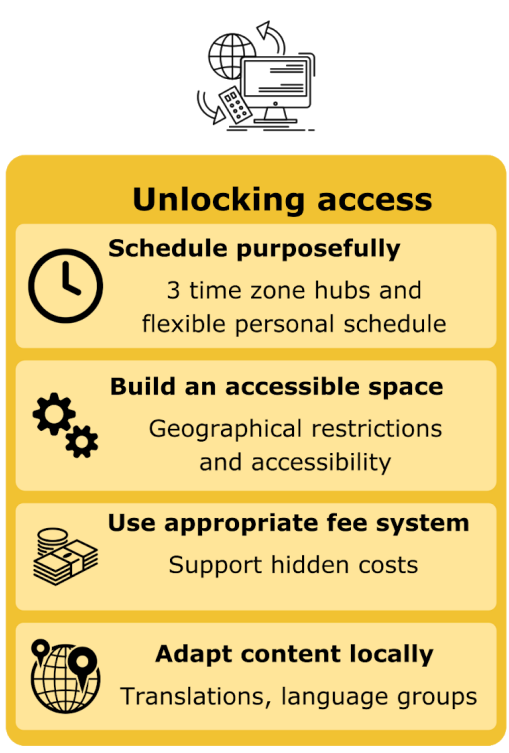

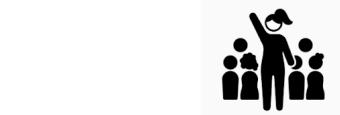

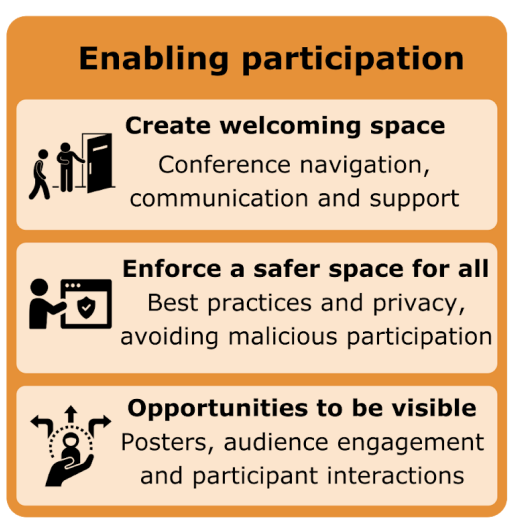

\section{Embracing open leadership}

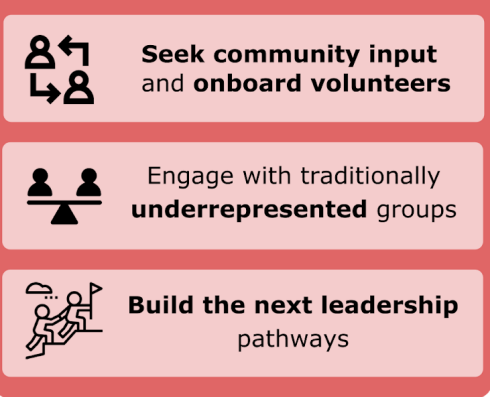

Fig. 1: Three steps (with recommendations) to improve the inclusivity of online conference: $A /$ Unlocking access; $\mathrm{B} /$ Designing for meaningful participation; $\mathrm{C} /$ Embracing co-creation and open leadership.

Credits: access CC-BY Flatart; clock CC-BY Shmidt Sergey; cog CC-BY John T. Garcia; money CC-BY Verry, ID; globe CC-BY Juan Pablo Bravo; participation Public Domain; door open CC-BY Gan Khoon Lav; safer CC-BY Adrien Coquet; visibility CC-BY Nithinan Tatah, co-creation CC-BY monkik; underrepresented CB-BY Nicole Hammonds; input CC-BY Prithvi; leadership CC-BY Life Skill

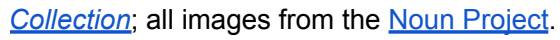

The Open Science Special Interest Group (OSSIG) of the Organization of Human Brain Mapping (OHBM) is dedicated to increasing open science practices in the brain mapping community. Each year this group holds two events: the OHBM Brainhack, which is a three-day hackathon, and the Open Science Room (OSR), a space for collaborations and talks within the organization's annual conference. In 2020, both events ran fully online for the first time and gathered over 1000 participants.

Here, by reflecting on our own practices and building on existing work, we survey and provide guidelines for enhancing inclusivity in online conferences (Fig. 1.). Although we have involved a diverse group of 
researchers in writing this paper, we recognise that there are many diverse perspectives and experiences that are not discussed here. This paper is organized in three sections. First, we focus on the mandatory step of removing barriers that prevent access to online conferences (A). We then discuss how to build an environment that will enable meaningful participation of a diverse audience (B). Finally, we look at the future and focus on building diverse leadership (C).

\section{A. Unlocking access}

\section{Schedule purposefully}

A.1.1 Three time zone hubs for global accessibility: Asia Pacific; Europe, Middle East and Africa; The Americas

There is no common 4-hour time range that can suit everyone across the globe (Fig. 2). Scheduling choices will inevitably determine a more local or global reach of the conference and may prioritize certain parts of the world.

Defining three time zone hubs_Asia, Pacific; Africa, Europe and Middle East; The Americas—and repeating the content at suitable times for each hub allows for content to be available nearly everywhere during typical working hours (for example OSR $2020^{1}$, NMA 2020). The repeated content can be interspersed with time blocks of hub-specific content such as questions to the speaker (Q\&A) and socials. To accommodate non-traditional schedules, each hub can remain accessible to any participant from any time zone.

To avoid having to rely on presenters giving their virtual talk three times, we encourage giving them the option of recording the talk in advance as well. Pre-recorded talks were also preferred by many conferences to avoid technical issues. Scheduling a time to play the recorded talks for each hub is useful for participants to interact with the speakers and other attendees. For Q\&A sessions, we recommend asking the presenters to nominate a representative (for example a collaborator) in each hub to participate in the Q\&A session. We also recommend grouping Q\&A sessions for multiple talks so as to make sure at least some of the talks will have speakers or representatives available for live Q\&A. In addition, there should be an option for asynchronous Q\&A in which written or recorded questions can be answered across a time range that is accessible to all participants.

While the three hub model is the most equitable solution, it is also more demanding for presenters and the organizing team. A less demanding option is to adopt a rotating schedule or very long days in order to

\footnotetext{
${ }^{1}$ All conferences cited in the manuscript are listed in Table 1 with their full name and website.
} 
prioritize one of those three geographical hubs for a subset of the conference (for example OHBM 2020). Finally, some conferences have decided to use a fully decentralized program (for example Brainhack Global 2020) or a single time zone with a more local reach (for example SIPS 2020) (see Fig. S1).

\section{A.1.2 Support the creation of flexible personal scheduling}

Different people have different time constraints. For example, caregivers will often not be available outside of typical working hours. Other professional groups who typically perform shift work may instead prefer to have content outside of 9-5 schedules to better balance professional responsibilities. A further consideration is those for whom extended periods of 'desk work' is physically or mentally prohibitive. As different groups may have contradictory preferences, we recommend taking into account potential attendees when making scheduling decisions. In general, we encourage online event organizers to explicitly prioritize attendee scheduling constraints in designing conference programs and carefully consider which days are included in the schedule. As online events incur no rental costs for a physical space, we suggest that longer events--free from the need to compress the content into a few days--may be better suited for some online events.

Regardless of the timing of the event, all attendees are more likely to be interrupted by their usual personal and professional routines when attending an online conference compared to the protected time in-person conferences can offer. Attendees with caregiving responsibilities may be especially likely to have an interrupted online conference experience. Many in-person conferences have provided childcare grants in the past few years to support parents in covering the costs of additional childcare, or to bring a relative to the conference (for example [6]). When shifting to virtual settings, some conferences have stopped offering dependent support—effectively suggesting that caregivers at home should be able to juggle their caregiving responsibilities with attending the conference. We strongly encourage event organizers to continue providing those grants and, in fact, extend them to all types of caregivers and not exclusively to parents. Those grants can be key to provide more flexibility to participate in the online event and are also more easily used at home (for example, participants can more easily request the help of a babysitter they trust or invite a parent to stay at home and help).

Incorporating breaks and providing the ability to watch talks, read post-talk discussions, and read live chat asynchronously makes it possible for attendees who cannot watch the content live to catch up and participate in the discussions. Furthermore, a less dense schedule can also help individuals to rest between events, without being forced to skip part of the content. This can be especially important for those who require additional accommodations. 
Hub 1: Asia, Pacific

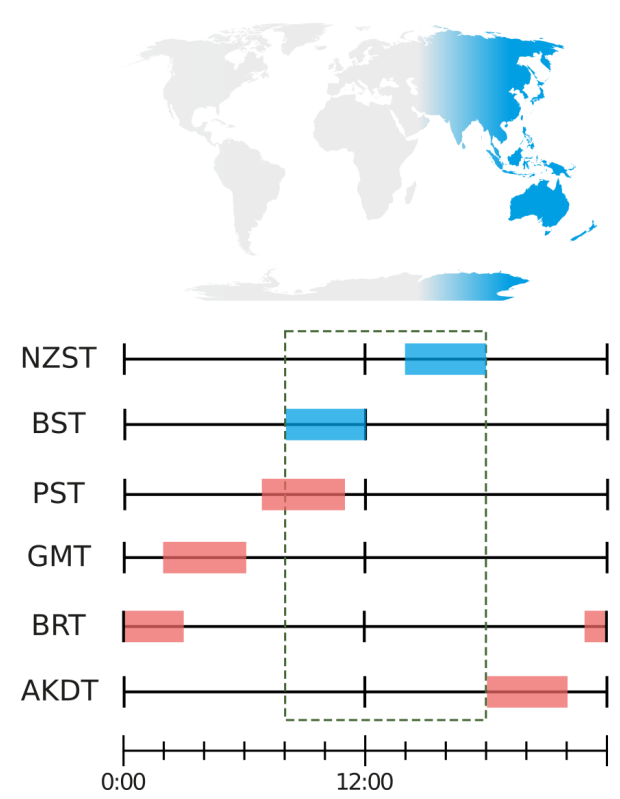

Hub 2: Africa, Europe, Middle East

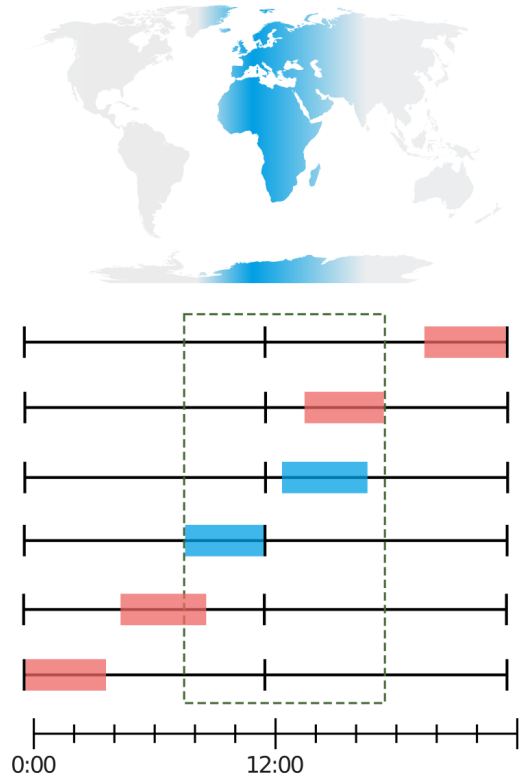

Hub 3: Americas

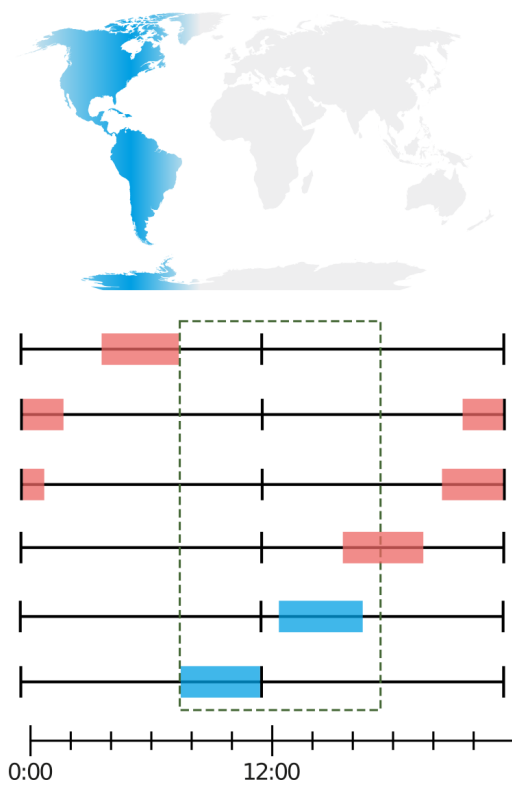

Fig. 2. Three time zone hubs for global accessibility: Asia Pacific; Africa, Europe and Middle East; The Americas. The coloured bars represent the schedule range of each hub in six representative time zones. For each time zone, a blue box indicates the timing corresponding to the recommended hub. For example, researchers based in New Zealand (NZST) can attend hub 1 during 14:00-18:00 (local time). They will be joined in this hub by researchers from Bangladesh (BST) for whom the content will happen 8:00-12:00 (local time). The green dashed rectangles cover typical working hours which often fall within the 8:00-18:00 range. The three time zone hubs make it possible to schedule 4 hours of content each day while remaining within typical working hours for nearly all time zones. NZST = New Zealand Standard Time (UTC+12); BST = Bangladesh Standard Time $($ UTC +6$) ;$ PST $=$ Pakistan Standard Time $(\mathrm{UTC}+5) ;$ GMT $=$ Greenwich Mean Time (UTC+0); BRT = Brasília Time (UTC-3); AKDT = Alaska Daylight Time (UTC-8).

\section{Build an accessible space}

\section{A.2.1 Identify geopolitical restrictions}

Online conferences rely on technical solutions to make their content available to attendees: conference website; videoconferencing; messaging systems; video broadcasting, etc. When selecting tools, review any geopolitical restrictions that would prevent access from certain regions of the globe (for example Youtube is not accessible from China). When a single solution cannot answer all needs, consider proposing alternative tools. For example, the OHBM Brainhack and OSR 2020 proposed the following alternative tools for users based in China: HackMD or Microsoft 365 (in place of Google Docs) for collaborative editing, WeChat (in addition to Twitter) for general announcements, DouYu (in addition to YouTube) for live broadcasting, and Bilibili (in addition to YouTube) to archive videos. Geopolitical restrictions can also apply when the host country has sanctions against a country whose citizens are potential conference attendees. This was experienced recently in preparation for the Neuromatch Academy [7], where volunteers were initially prevented from providing educational services to attendees from Iran, due to sanctions imposed by the US government [8]. 


\section{A.2.2 Select tools with consideration of disability accommodations}

When selecting technical solutions, it is important to review their accessibility for individuals with disabilities. A first step is to ensure that the chosen solutions comply with existing accessibility standards, are compatible with adaptive technology (such as screen readers) and enable the addition of closed captions $($ see $[9,10]$ for recent reviews of the accessibility of conferencing solutions). When possible, we recommend using professional subtitles. While automatic subtitles are a cheaper option and have made great progress in the past few years (see [11]), they can unfortunately reinforce disparities among speakers as content is often better recovered for those who are native speakers. Providing a textual version of all audio content will not only help those whose vocal understanding is impaired and those experiencing poor sound quality but will also make it easier for non-native English speakers to follow along. Transcripts can also serve as input for all existing text-based accessibility features, such as automated text-to-speech or translation and therefore extend the accessibility of the content to those who use those technologies. In all cases, accessibility features should be turned on as standard, rather than waiting for them to be requested by the individuals who need them.

\section{A.2.3 Solicit and act upon feedback}

Especially when solutions may not be obvious, it is important to provide participants - at an early stage - with ways to provide input or submit a request for additional solutions that meet their needs. Consider how to make the request for assistance as little of a burden to the participant as possible and avoid using stigmatizing language. A plan for how these needs will be accommodated should be created, including a backup plan in the case that needs fall outside of the organizers' means. To this end, it may be helpful to consult with institutional or organizational disability and accessibility experts for each unique group that may require additional support.

\section{Don't let the cost of the conference be a barrier for attendance}

\section{A.3.1 Define an appropriate fee system}

Online conferences offer a unique opportunity to broaden geographical diversity, but fees can remain a significant barrier if they do not take into account differences across countries. We recommend setting multiple fee levels by taking into account both the level of income (for example [12]) and the support provided to scientific research (for example [13]). In addition, we recommend providing a mechanism to waive fees for 
attendees to account for variations within countries and for individual circumstances such as no or less institutional support (OSR 2020, Neuromatch Academy 2020).

In many countries, additional fees and even taxes are incurred when paying with non-local currencies; we recommend using a platform that allows for payment in local currencies so as to not burden individual participants with additional fees. The use of credit cards is also not ubiquitous everywhere. For example, some countries mainly use cash, and others have local electronic payment systems. As some participants might not have access to a credit card, it is important to support multiple payment methods. Options include allowing mobile money payments or app-based payment platforms, which can be connected directly to a bank account. In tandem, consider an option for lab or group fees for online events as these can help reduce administrative burden and ensure that more early career trainees and support staff can attend.

Finally, as online conferences often cost less than their in-person counterparts, this is also the opportunity to consider reducing the fees overall (for example NeuroHackademy 2020 was offered gratis).

\section{A.3.2 Provide support for hidden costs}

Online conferences create new technical requirements for attendees. A combined headset or a desktop microphone and a set of headphones can help create a more immersive experience or even enable participation. Part of the minimal requirements is also access to a high-quality, high-speed internet connection to support video-based systems, or, at the very least, a connection capable of supporting consistent text-based communication in various messaging systems [14].

Consider reducing the amount of live connection time and required bandwidth. While live-streaming is a very popular feature as it enables direct interaction, it often requires a high-bandwidth internet connection. Provide an option to downgrade the quality of the video (or completely switch it off) and the possibility to download content rather than requiring participants to live-stream it. For content that can be watched later, provide the possibility to watch at a different speed. It is also preferable to make recordings of content available as soon as possible after live events, so those watching offline can contribute to the live text-based discussion.

Although there are several ways to reduce the barrier of hidden costs to access, we are especially interested in modes which synergistically increase accessibility in multiple domains. For example, if speakers are pre-recording their talks for presentation in different hubs (see: A.1, Schedule purposefully), providing an option to record the talk without being connected is especially important to ensure high-quality recordings for 
those with an unstable internet connection. We also encourage adapting existing infrastructure from in-person events to support online experiences. For example, in place of travel grants, consider offering small grants to offset the cost of small equipment (headphones, microphone, webcam) or a temporary SIM card to boost connectivity (for an example see OpenMR Virtual 2021).

\section{Adapt the content to local audiences}

As for in-person conferences, the provision of English-only content is a significant hurdle to many participants around the world [15] and online conferences offer an opportunity to lower this barrier.

Organizers can encourage the formation of connections between individuals speaking the same language to promote networking and communication for individuals less fluent in English (Neuromatch Academy 2020) [7]. This might be facilitated through the formation of local gatherings, which can also organize live translation with a professional using a separate audio channel (TPATH 2021). Part of the program can be delivered in other languages with a large audience of speakers such as Spanish or Chinese (for example OHBM Brainhack 2020).

Subtitles and closed captions can be included in different languages. In the case of pre-recorded content, this can be incrementally improved by adding crowd-sourced closed captioning in multiple languages (for example PyCon 2019). When possible, consider supporting the creation of local gatherings that will provide facilities so that individuals can attend the conference as a group [16] with the added benefit to support local interactions and networking.

\section{B. Designing for meaningful participation}

\section{Create a welcoming space}

\section{B.1.1 Design navigation through the online conference}

Navigating an online conference can be a new and sometimes frustrating experience. Researchers who have attended online conferences may struggle with a new platform given the variability in available structures and software, and first-time conference attendees might be especially unclear on what to expect if they are not already familiar with the in-person equivalent of the meeting. This difficulty has been amplified by the rapid transition to virtual conferencing since there were relatively few pre-existing industry standard platform options, resulting in a large variety of solutions. 
To facilitate this experience, organizers should design user-friendly navigation through the online conference. This starts with the definition of what the "entrance" will be and how attendees will go from one space to another. When doing so, consider how different users will connect and participate, for example based on geographical location, or whether the content is watched live or asynchronously. We would recommend summarizing this information as a "cheat sheet" that describes the best places to go for different types of interactions.

It is equally important to plan how and when connection information will be shared. For in-person conferences, the address of the venue is easily shared, but online conferences provide unique challenges in publicizing the event. Specifically, many conference organizers may prefer to avoid posting connection details publicly to limit the risk of malicious participation, i.e. any non-affiliated attendee joining with the intent to disrupt or otherwise derail the event. Because connection details may not be posted publicly, a backup plan for attendees who did not get connection information (for example the connection details were blocked by an email filter) in time should be easily accessible. Contact details for support on connection or technical issues should be easily accessible and posted in multiple locations.

In addition, we recommend repeating the connection links on the program, with only the password or login information being passed privately to each attendee. Provide a schedule in local time for each attendee or, if this is not possible, pick Universal Time Coordinated (UTC) over a local time zone (see BCW 2021).

\section{B.1.2 Communicate and provide training}

In order to accommodate all levels of digital literacy and familiarity with digital tools and platforms, instructions about the software and tools should be made available a few weeks before the conference along with an interactive demo of the platform. Giving attendees the option to connect early on may potentially identify issues ahead of time, and a complete tour of the various online tools can be provided prior to the start of the conference. Each session of the conference should start with a quick overview of the main ways to participate and interact with the content.

It can be particularly stressful for attendees to keep track of very long emails describing all available tools, and we recommend sharing only as much information as needed at a time while reserving more detailed information for a frequently asked questions page on your conference website. Similarly, we encourage conference organizers to establish which communication channels will be used to contact attendees, speakers and volunteers during the event (for example email versus conference platform) and disseminate specific types of information ahead of time. In general, it is best to avoid last minute changes in technology 
as these are likely to disproportionately impact attendees who are less connected with other members of the community, such as first-time attendees.

\section{B.1.3 Provide on-site support}

During an online conference it is often more difficult for less experienced attendees to rely on their more experienced peers to guide them through the event. This difficulty can be mitigated by building a support system to help orient and guide attendees in the virtual conference. Some conferences have positioned greeters at their entrances, or run an all-day "welcome desk" (as a videoconference or chat room) where attendees could find facilitators and ask questions as needed. Other conferences have connected attendees through a "buddy system", pairing first-time attendees with more experienced participants. This should be distinct from the support handling connection or technical issues.

\section{Promote and enforce a safer space for all}

\section{B.2.1 Follow best practices of inclusive in-person conferences}

To provide a safer online environment, it is important to adapt best practices applied to inclusive in-person conferences. One can minimise the risk of microaggression being portrayed by speakers, attendees, or through shared scientific content by having an explicit code of conduct (for example OHBM 2020 Code of Conduct [17]) in place. To be effective, the code of conduct must be acknowledged and accepted by all participants (for example, as part of the registration) and must include dedicated procedures to enable reporting and dealing with violations. To make enforcement possible, official conference forums where unmoderated interactions between attendees are possible should require user credentials and ways to exclude individuals who violate the code of conduct across all conference sites. If a code exists already for a given community, it should be adapted to the online context. If the event is global, it is important to ensure that reporting can be done efficiently from all time zones and in multiple languages, with the help of a globally distributed committee.

To provide an opportunity for researchers to self-identify their gender, conference organizers should endorse and normalize the use of pronouns [18]. This is especially necessary in online environments where gender as a social construct may not be as easily expressed. Pronouns can for instance be added to biography or display names on the various platforms, and all participants should be encouraged to specify their pronouns to normalize this practice. 


\section{B.2.2 Reduce the risk of malicious participation}

Online conferences are at risk of a new kind of aggressive behaviour: malicious participation, colloquially referred to as "zoom bombing", in which bots or anonymous spammers enter the event and take control of the audio or video channels to share unwanted material (see section B.1.1). A number of best practices have been proposed to reduce the risk of this, including the adoption of registration processes that make it possible to share information only with those registered (even if the event is free), or the use of software that allows for administrative control of who can speak and share screen (for more see [19]). In addition, we recommend preparing a crisis plan in case this form of aggression does happen, including a strategy for removing the intruder and clarifying who has the responsibility to do so.

\section{B.2.3 Explicitly consider data privacy}

Data protection and privacy in an online conference is both an ethical and a legal concern. With content being recorded and broadcasted, online conferences increase participants' exposure and require organizers to think carefully about data privacy [19]. It is important to be explicit about what is recorded or broadcasted, and obtain the consent of participants before sharing any of their personal content in compliance with regulations such as the European General Data Protection Regulation. Provide different ways to participate beyond joining on-screen (for example, text chat, shared collaborative notes), and consider the data privacy policies of the tools used (for example which data is stored, its persistence, and whether it will be used by the company or shared with others). Conference organizers should ensure that the collection, use, and disclosure of personal data and, in particular, their likeness is consistent with the expectations of attendees, and communicate these details clearly.

\section{Provide opportunities to increase the visibility of all participants}

In the rush to convert in-person conferences into online events, most of the attention has been given to online talks, effectively skewing online conferences to give more visibility to already established researchers. In this section, we review ongoing efforts into translating other conference features. Beyond the options discussed here, many solutions are still to be found or adopted [20] and we encourage online conference organizers to think creatively about how best to deliver their content.

\section{B.3.1 Design interactive poster sessions}

Scientific posters have a long-standing tradition in academic conferences and are more often presented by early career researchers than those with established research careers. Relieved from the constraints of a 
physical space, online conferences may offer an opportunity to broaden the set of presenters who are selected to display their work and possibly increase the duration during which posters are displayed. But with more content available, online conferences are also more difficult to navigate. A number of attempts have been made in the past year to reproduce and extend poster sessions in an online setting.

Some conferences transformed online poster sessions into a series of very short talks in front of the whole audience. While those short talks are a good way to provide an overview of a large number of posters in a short amount of time, they do not alone replace the long explanations, reserved to an exclusive but also potentially random audience which characterize poster sessions and that very often translate into collaborations. To this aim, some conferences have successfully used map-based applications that mimic a physical space in a virtual environment (for example Gather [21] was used at Cosyne 2020). This provides a spatial familiarity to conference interactions. Since it is harder to stumble upon an interesting poster online, a well-designed abstract search system, as well as mechanisms to create and share curated lists will bring a greater number of abstracts to the attention of potentially interested attendees. Similar to the earlier recommendations for talks (see section A.1.1), a solution should be available to ask and answer questions about a poster asynchronously.

\section{B.3.2 Provide multiple opportunities for audience engagement}

Online conferences offer new opportunities for participant engagement. Proposing different channels with video, audio-only, or text-based methods [11] can reduce the barriers for asking a question, and designated volunteers can post on behalf of participants who would prefer to ask a question anonymously (for example in ABCD-ReproNim). Solutions are also available to let the audience vote for comments they like and steer the conversation towards topics of common interest. In addition, a live chat function also creates a sense of community as the audience can discuss the presentation, ask shorter clarifying questions, or post relevant literature to everyone. The chat also allows the speaker to receive feedback and applause from the audience, making a potentially impersonal online talk more rewarding and stimulating. Additionally, audience interaction solutions can be used to allow speakers to engage with their audience. At in-person conferences, longer discussions with the speaker are often held privately after the talk has ended. While this discourse often happens spontaneously at in-person conferences, in online conferences, the communication channel that will make this exchange possible has to be actively planned and implemented. More generally, the selected platform should offer the possibility to schedule impromptu meetings in a given location at a given time for follow-up discussions. 
Moving from in-person meetings to virtual settings also offers exciting opportunities to advance beyond the traditional knowledge delivery format of the monologue lecture followed by a question session. Looking at non-academic consumption of information, it is apparent that attention may be better retained when switching between different speakers (as is used in the majority of news programs), or by using an interview format that mimics natural interaction even when it is not live (as is used in many podcasts), or through the use of video graphics (as is used in documentaries and instructional videos). We encourage online conference organisers to think creatively about the potential of these alternative modes of delivery.

\section{B.3.3 Promote participant-to-participant interactions}

A central aspect of in-person conferences are not only the talks and posters, but also the unplanned encounters among attendees. These are especially important for early career researchers, who may depend on these connections for collaboration and career opportunities. Online conferences will benefit from actively promoting opportunities for such encounters by providing planned social events and areas for attendees to mingle in between scheduled sessions.

A major challenge for online events is to motivate community members to socialize without access to typical in-person conference incentives such as food and in-person activities. Some conferences have used a gamified videoconferencing platform (See section B.3.1, for example ISMRM 2020 used gather town for their newbie reception) that allows spatially determined video interactions between attendees as well as an array of engaging activities, game rooms, or an art gallery (for instance the BIIM 2020 organized a scavenger hunt). Other platforms encourage users to interact by assigning points to activities such as posting on a message board, generating competition between participants to accrue points on a publically visible leader board of engagement.

Beyond planned social activities, strategies for promoting online interaction and networking are an emerging topic; depending on the funds available one might also consider screening movies, having food or items delivered (best for small groups), and grouping or pairing individuals for meetings (NeuroMatch).

\section{Embracing co-creation and open leadership}

Beyond increasing the representation of participants and speakers, inclusive online conferences need to provide opportunities for all to influence decisions about the conference and to join leadership. 


\section{Onboard volunteers and seek community input}

Expanding on the idea of reaching out to attendees to help define the conference schedule (see section $A$ 1.2.), soliciting input of potential future participants is a great way to build a conference that meets the expectations of the community. Onboarding volunteers and proposing various ways of being involved, with roles requiring different levels and types of commitment, not only lowers the barrier to engagement but provides an active form of participation for new community members. Different roles may involve the same total time commitment but vary with regard to how the work needs to be scheduled, e.g. moderating a session for two hours straight versus spending two hours maintaining a conference website over the course of a week. It is important to advertise those roles early and often across multiple venues and provide comprehensive guidelines for volunteers and clear assignment of responsibilities. This may not only help relieve imposter syndrome for newly recruited volunteers, but it will give team members freedom and confidence to complete their tasks effectively.

Studies have shown that marginalised researchers are more likely to innovate in ways that might not be apparent to majoritarian reviewers and to cross disciplinary boundaries more or differently than their majoritarian peers [22]. Inclusivity should not only apply to the conference setup but also to its content. This idea - termed epistemic inclusivity - calls for the inclusion of multiple lines of enquiries, broad perspectives, contradictory views, and even different scientific paradigms, such that a multiplicity of theories and hypotheses can be formulated and discussed instead of only the dominant ones.

\section{Engage with underrepresented groups from the start}

In the active process of allowing attendees to have more of a voice in all aspects of the conference (see section A.2.3), it is important to specifically seek out underrepresented voices. By definition, members of underrepresented groups will not be well-represented in input provided by the community. Being intentional about seeking this input, listening, and including these perspectives is essential to successfully accommodate a diverse audience. As with traditional conferences, involving underrepresented groups in the formation of organizing committees, speaker selection, sharing of announcements, or the recruiting of volunteers promotes researchers in these groups implicitly into positions of authority. Ensuring that the organisation promotes underrepresented researchers with work that is a part of routine responsibilities, rather than by heaping unpaid labour upon those who are already disadvantaged, will promote their sustained involvement. Members of the most underrepresented groups are often the most difficult to find and recruit for an event, and it is possible that when found they may have limited time because they are already 
serving as a representative of their group in other forums. When soliciting these groups, consider not only waiving attendance fees, but also paying honoraria to speakers and organisers in exchange for their labour.

A number of groups were created in the past few years to highlight the diversity of our research community. In neurosciences those include: Black in Neuro [23], The Women in Neuroscience Repo [24], Queer in Neuro [25], Latinx in Neuro [26], and Disabled in STEM [27], Innovators in Cognitive Neuroscience [28]. Involving these communities and following the resources they have created is essential to benefit from their knowledge and diversify scientific spaces both in terms of the research that is highlighted and to improve scientific culture.

\section{Create pathways to join leadership}

Online conferences are in their infancy, and the scientific community has an opportunity to create new formats that will enable more diverse participation. Future online conferences will only be more inclusive if their leadership is more diverse and it is essential to create opportunities now in order to diversify leadership in the coming years. Developing contributor pathways to specify how people can progress from attending the conference into joining leadership roles, can both provide transparency on how leadership roles can be reached and help identify barriers to a diverse leadership (see [29] for more open leadership practices).

\section{Conclusions}

Online conferences offer unique opportunities to promote a more diverse scientific community. Some international organizations have already announced considering hybrid online and in-person conferences for the future, and the shift initiated in 2020 is likely to impact research communities durably. We offered recommendations to center inclusivity in the design of online conferences to purposefully encourage a diverse community to join, to actively participate in, and to lead online conferences. Online conferences are just at their infancy, and we expect that many aspects will evolve and improve in the future as communities take ownership of this new format. We also believe that making conferences more inclusive should go hand in hand with addressing larger structural issues - such as systemic racism and sexism. As an example, the widespread practice of relying on unpaid labour to set up and run conferences is especially problematic, and we hope that in the future appropriate compensations can be designed for those who invest their time and energy in organizing scientific events alongside their other professional commitments. We believe that the global challenges we are facing during the COVID-19 pandemic, taken together with recent events in different countries with respect to marginalized groups, should be a wake-up call for the scientific community to work towards the advancement of more inclusive conferences. The suggestions provided here reflect our 
shared experience of building a conference to serve our community, as a group of research professionals with a commitment to improving inclusivity in our field. We acknowledge, however, that these concerns in many ways reflect the diversity of our own leadership, which is notably lacking in some aspects. Accordingly, we expect and hope that these suggestions are not an exhaustive account of practices necessary to build a fully accessible and inclusive conference, but rather a mechanism to signpost and learn from the experiences of 2020, making space for event organisers to focus on further improvements for 2021 and beyond.

Table 1: Examples of online conferences cited in the main text.

\begin{tabular}{|c|c|c|}
\hline $\begin{array}{l}\text { Conference } \\
\text { abbreviation }\end{array}$ & Full name & Website \\
\hline ABCD-ReproNim & $\begin{array}{l}\text { ABCD-ReproNim: Reproducible Analyses of } \\
\text { ABCD Study Data }\end{array}$ & https://www.abcd-repronim.org \\
\hline BCW 2021 & The Brain Connectivity Workshop 2021 & https://bcw-2021.com \\
\hline BIIM 2020 & BRAIN Initiative Investigators' Meeting 2020 & https://www.brainmeeting2021.com/ \\
\hline Brainhack Global 2020 & - & https://brainhack.org/global2020/ \\
\hline Cosyne 2020 & $\begin{array}{l}\text { Computational and Systems Neuroscience } \\
2020\end{array}$ & $\begin{array}{l}\text { http://www.cosyne.org/c/index.php?title= } \\
\text { Cosyne } 21\end{array}$ \\
\hline ISMRM 2020 & $\begin{array}{l}\text { Annual Meeting of the International Society } \\
\text { for Magnetic Resonance in Medicine } 2020\end{array}$ & https://www.ismrm.org/20m/ \\
\hline NeuroHackademy 2020 & - & https://neurohackademy.org/ \\
\hline NMA 2020 & NeuroMatch Academy 2020 & https://www.neuromatchacademy.org/ \\
\hline OHBM 2020 & $\begin{array}{l}\text { Annual Meeting of the Organization for } \\
\text { Human Brain Mapping } 2020\end{array}$ & $\begin{array}{l}\text { https://www.humanbrainmapping.org/i4a/ } \\
\text { pages/index.cfm?pageid=3958 }\end{array}$ \\
\hline OHBM Brainhack 2020 & $\begin{array}{l}\text { Brainhack of the Organization for Human } \\
\text { Brain Mapping } 2020\end{array}$ & https://ohbm.github.io/hackathon2020/ \\
\hline OpenMR Virtual 2021 & - & $\begin{array}{l}\text { https://openmrbenelux.github.io/2021/pa } \\
\text { ge-program/ }\end{array}$ \\
\hline OSR 2020 & Open Science Room 2020 & https://ohbm.github.io/osr2020/ \\
\hline PyCon 2019 & Python Conference 2019 & https://us.pycon.org/2019/about/ \\
\hline SIPS 2020 & $\begin{array}{l}\text { Society for the Improvement of Psychological } \\
\text { Science } 2020\end{array}$ & $\begin{array}{l}\text { https://www.improvingpsych.org/SIPS20 } \\
\underline{\underline{20 /}}\end{array}$ \\
\hline TPATH 2021 & $\begin{array}{l}\text { Transgender Professional Association for } \\
\text { Transgender Health } 2021\end{array}$ & https://tpathealth.org/conference2021/ \\
\hline
\end{tabular}

The conferences are listed with their abbreviated name and year, their full name and their website. 


\section{References}

1. Sarabipour S. Virtual conferences raise standards for accessibility and interactions. eLife. eLife Sciences Publications, Ltd; 2020; doi: 10.7554/eLife.62668.

2. Skiles M, Yang E, Reshef O, Muñoz D, Cintron D, Lind ML, et al.. Beyond the carbon footprint: Virtual conferences increase diversity, equity, and inclusion. 2020; doi: 10.21203/rs.3.rs-106316/v1.

3. Singleton KS, Tesfaye R, Dominguez EN, Dukes AJ. An open letter to past, current and future mentors of Black neuroscientists. Nat Rev Neurosci. Nature Publishing Group; 2021; doi: 10.1038/s41583-020-00421-9. 4. Niner HJ, Wassermann SN. Better for Whom? Leveling the Injustices of International Conferences by Moving Online. Front Mar Sci. Frontiers; 2021; doi: 10.3389/fmars.2021.638025.

5. Whitaker K, Guest O. \#bropenscience is broken science. :42020;

6. Tzovara A, Amarreh I, Borghesani V, Chakravarty MM, DuPre E, Grefkes C, et al.. Embracing diversity and inclusivity in an academic setting: Insights from the Organization for Human Brain Mapping. PsyArXiv; 2020 Oct.

7. van Viegen T, Akrami A, Bonnen K, DeWitt E, Hyafil A, Ledmyr H, et al.. Neuromatch Academy: Teaching Computational Neuroscience with global accessibility. ArXiv201208973 Q-Bio. 2020;

8. Ro C. How researchers overturned US sanctions on a virtual summer school. Nature. Nature Publishing Group; 2020; doi: 10.1038/d41586-020-02347-9.

9. Hare L: Accessibility at useR! 2021. https://user2021.r-project.org/participation/accessibility/ (2021). Accessed 2021 Mar 19.

10. World Blind Union: Accessibility : World Blind Union. https://worldblindunion.org/resources/accessibility/ (2020). Accessed 2020 Nov 27.

11. Yehudi Y, Whitney KS, Sharan M. Enhancing the inclusivity and accessibility of your online calls. 2020; doi: 10.31219/osf.io/k3bfn.

12. World Bank: World Bank Country and Lending Groups - World Bank Data Help Desk.

https://datahelpdesk.worldbank.org/knowledgebase/articles/906519-world-bank-country-and-lending-groups (2021). Accessed 2021 Feb 22.

13. UNESCO Institute for Statistics: How much does your country invest in R\&D?

http://www.uis.unesco.org/_LAYOUTS/UNESCO/research-and-development-spending/index-en.html (2021). Accessed 2021 Feb 22.

14. Garrity J. The State of Broadband 2020: Tackling digital inequalities - A decade for action. ITU/UNESCO Broadband Commission for Sustainable Development; 2020.

15. Perez R: Science's English dominance hinders diversity—but the community can work toward change. Sci. AAAS.

https://www.sciencemag.org/careers/2020/10/science-s-english-dominance-hinders-diversity-community-can -work-toward-change (2020). Accessed 2020 Nov 17.

16. Reshef O, Aharonovich I, Armani AM, Gigan S, Grange R, Kats MA, et al.. How to organize an online conference. Nat Rev Mater. Nature Publishing Group; 2020; doi: 10.1038/s41578-020-0194-0.

17. Organization for Human Brain Mapping: Code of Conduct.

https://www.humanbrainmapping.org/i4a/pages/index.cfm?pageid=3912 Accessed 2021 Apr 30.

18. Cooper KM, Auerbach AJJ, Bader JD, Beadles-Bohling AS, Brashears JA, Cline E, et al.. Fourteen

Recommendations to Create a More Inclusive Environment for LGBTQ+ Individuals in Academic Biology.

CBE_Life Sci Educ. American Society for Cell Biology (Ise); 2020; doi: 10.1187/cbe.20-04-0062.

19. ACM: Report on Virtual Conferences. https://www.acm.org/virtual-conferences (2020). Accessed 2020

Nov 17.

20. Woodley L, Pratt C, Ainsworth R, Amsen E, Bakker A, Butland S, et al.. A guide to using virtual events to facilitate community building: event formats. Zenodo; 2020; doi: 10.5281/zenodo.3934385.

21. Gather: Better spaces to gather around. https://gather.town/ Accessed 2021 Apr 30.

22. Hofstra B, Kulkarni VV, Galvez SM-N, He B, Jurafsky D, McFarland DA. The Diversity-Innovation

Paradox in Science. Proc Natl Acad Sci. National Academy of Sciences; 2020; doi:

10.1073/pnas.1915378117.

23. Black in Neuro: Black in Neuro. Black Neuro. https://www.blackinneuro.com Accessed 2021 Apr 30.

24. Schrouff J, Pischedda D, Genon S, Fryns G, Pinho AL, Vassena E, et al.. Gender bias in (neuro)science: Facts, consequences, and solutions. Eur J Neurosci. 2019; doi: 10.1111/ejn.14397.

25. Queer in Neuro: @QueerInNeuro. Twitter. https://twitter.com/QueerInNeuro Accessed 2021 Apr 30.

26. Latinx In Neuro: @LatinxInNeuro. Twitter. https://twitter.com/LatinxInNeuro Accessed 2021 Apr 30.

27. Disabled In STEM: Inclusivity For All. DisabledInSTEM. https://disabledinstem.wordpress.com/ Accessed 2021 Apr 30.

28. Innovators in Cognitive Neuroscience: Pioneering ideas of the human mind.

https://innovatorsincogneuro.github.io/ Accessed 2021 Apr 30.

29. Mozilla: Open Leadership Framework.

https://mozilla.github.io/open-leadership-framework/framework/\#what-is-open-leadership (2018). Accessed 


\section{Supplementary}

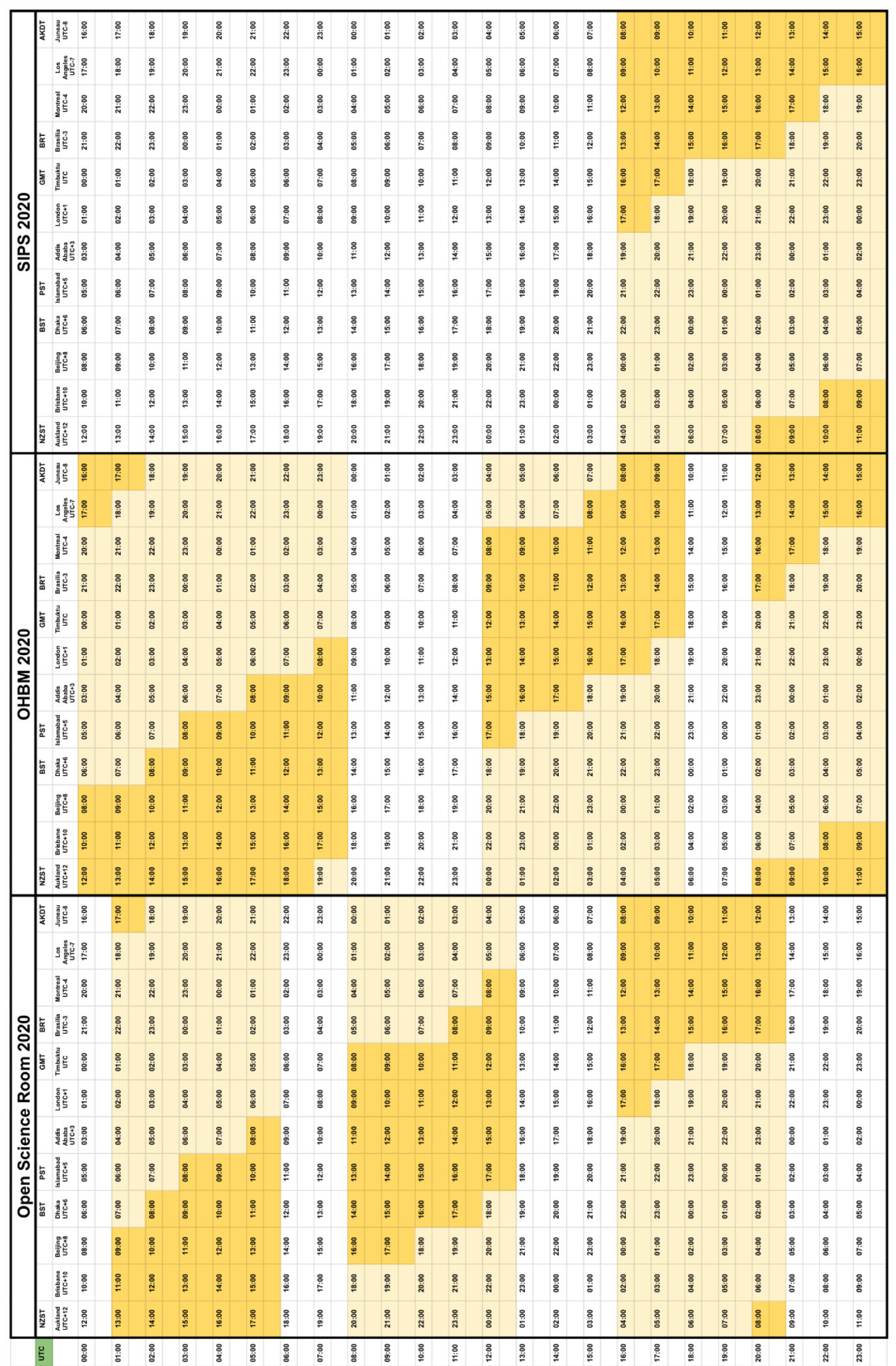

Fig. S1: Comparison of the schedules of OSR 2020, OHBM 2020 and SIPS 2020. The light yellow background represents the schedule range for each conference with darker yellow corresponding to parts of the schedule that may overlap with typical working hours, which often fall within the 8:00-18:00 range. 


\section{Declarations}

The presented opinions are those solely of the authors and do not necessarily represent the opinions of the National Institutes of Health.

\section{Ethics approval and consent to participate}

Not applicable.

\section{Consent for publication}

Not applicable.

\section{Data Availability}

Not applicable.

\section{Competing interests}

A.E.V is currently employed by the commercial company Figshare. Support from this employer was provided in the form of the author's salary, but the employer has not influenced the development of this project nor the decision to publish this work.

\section{Funding}

C.G.v.P. received funding from the Medical Research Council UK and by the NIHR Oxford Health Biomedical Research Centre. A.R.L. and K.L.B. were supported by NIH R25-DA051675, NIH U01-DA041156, NSF 1631325, and NIH R01-DA041353. A.N. is supported by the Brain and Behavior Research Foundation NARSAD grant and NIMH grant 5R21MH118556-02. E.A-O. is supported by TransMedTech Institute fellowship. T.A. is supported by Biotechnology and Biological Sciences Research Council, London ([BB/S008314/1] (PI: Ines Violante). S.B. is supported by the National Imaging Facility, a National Collaborative Research Infrastructure Strategy (NCRIS) capability, at the Centre for Advanced Imaging, the University of Queensland. M.F. received funding from the European Research Council (ERC) under the European Union's Horizon 2020 research and innovation program (ERC Advanced Grant agreement No 694665: CoBCoM - Computational Brain Connectivity Mapping. PI: Rachid Deriche). M.G. is supported by the Elsass Foundation (18-3-0147). E.A.G.-V. is supported by the Laboratorio Nacional de Imagenología por Resonancia Magnética (LANIREM). T.G. is supported by a Marie Sklodowska-Curie Global Fellowship under 
the European Union's Horizon 2020 research and innovation programme. O.G. was supported by the Netherlands Organization for Scientific Research (Grant 016.Vidi.188.029) awarded to Dr. Andrea E. Martin. V.I. is supported by the MIUR project "Dipartimenti di eccellenza". D.B.K. was supported by the National Institute of Mental Health under grant RF1 MH120021. L. L.-P. was supported in part by the Translational Research Institute grant TL1 TR003109 through the National Center for Advancing Translational Sciences of the National Institutes of Health (NIH). A.L. is supported by the UK Medical Research Council (MR/N013700) and King's College London member of the MRC Doctoral Training Partnership in Biomedical Sciences. A.L. is supported by a PhD Studentship awarded from the Wellcome Trust (109062/Z/15/Z). M.M. is funded by the Wellcome Trust through a Sir Henry Wellcome Postdoctoral Fellowship [213722/Z/18/Z]. S.M.L. is supported by a Melbourne Research Scholarship. G.N. is supported by the AXA Research Fund. NeuroHackademy is supported through R25 MH112480 from the National Institute of Mental Health (PI: Ariel Rokem). T.C. is supported by the National Imaging Facility, a National Collaborative Research Infrastructure Strategy (NCRIS) capability, at Sydney Imaging, The University of Sydney. Supported in part by the Intramural Research Program of the NIMH: E.L by ZIAMH002949, D.H. by ZIAMH002783, D.M. by ZICMH002960, M.N. by ZIAMH002909. S.V.D.B. was supported by the Research Foundation Flanders, grant number: G036716N

\section{Authors' contributions}

E.L. and R.G. chaired the 2020 OHBM Brainhack, and C.G.v.P. and S.H. chaired the 2020 Open Science Room, the two online events that led to the writing of this review. Other members of the OHBM Open Science Special Interest Group (OSSIG) 2020 leadership were: C.M. (chair), E.D. (treasurer), A.E.V.G (secretary), K.B. (secretary elect), A.N. (chair elect), T.G. (treasurer elect), K.W. (past chair), E.Dup (past secretary), G.K. (past treasurer). All authors contributed to the OHBM Brainhack 2020 and/or the OSR 2020 as volunteers and/or speakers and/or were members of the OHBM OSSIG 2021 leadership. Figures: M.M, G.N. R.G., S.H., F.V., C.M. Writing of the manuscript: C.M, E.L., C.G.v.P., R.G., S.H. with support from all authors who reviewed the manuscript and contributed original ideas. The definition of "epistemic inclusivity" was contributed by P.D.M.

Contributions according to CASRAI CRediT taxonomy

\begin{tabular}{|l|l|}
\hline Conceptualization & $\begin{array}{l}\text { C.M, E.L., C.G.v.P., R.G., S.H. with support from all } \\
\text { authors who contributed original ideas. }\end{array}$ \\
\hline $\begin{array}{l}\text { Supervision, Project Administration, Funding } \\
\text { Acquisition }\end{array}$ & $\begin{array}{l}\text { E.L., C.G.v.P., R.G., S.H., C.M, E.D., A.E.V.G, K.B., } \\
\text { A.N., T.G. }\end{array}$ \\
\hline Writing - Original Draft Preparation & C.M, E.L., C.G.v.P., R.G., S.H. \\
\hline
\end{tabular}




\begin{tabular}{|l|l|}
\hline Writing - Review \& Editing & All authors \\
\hline Visualization & M.M, G.N. R.G., S.H., F.V., C.M. \\
\hline
\end{tabular}

\section{Acknowledgements}

In 2020, the OHBM Open Science Special Interest group was supported by Openneuro (https://openneuro.org/), INCF (https://www.incf.org/), Neuromod (https://www.cneuromod.ca/), CONP (https://conp.ca/), the Wellcome Centre for Integrative Neuroimaging (https://www.win.ox.ac.uk/), UNIQUE (https://www.unique.quebec/), QBIN (https://www.rbiq-qbin.qc.ca/Accueil), OHBM Australian chapter (https://ohbmaustralia.wordpress.com/) and OHBM (https://www.humanbrainmapping.org). We would also like to thank all the participants, speakers and volunteers of the 2020 Open Science Room and OHBM Brainhack. 